\title{
MODEL DEVELOPMENT AND CALIBRATION FOR THE COUPLED THERMAL, HYDRAULIC AND MECHANICAL PHENOMENA OF THE BENTONITE
}

\author{
M. Chijimatsu' ${ }^{1}$, L. Börgesson ${ }^{2}$, T. Fujita ${ }^{3}$, J. Hernelind ${ }^{2}$, P. Jussila ${ }^{4}$, T.S. Nguyen ${ }^{5}$, \\ J. Rutqvist ${ }^{6}$ and L. Jing ${ }^{7}$ \\ $\left.{ }^{1}\right)$ Hazama Corporation, Japan \\ 2) Clay Technology, Sweden \\ ${ }^{3}$ ) Japan Atomic Energy Agency, Japan \\ ${ }^{4}$ ) Helsinki University of Technology, Finland \\ $\left.{ }^{5}\right)$ Canadian Nuclear Safety Commission, Canada \\ ${ }^{6}$ ) Lawrence Berkeley National Laboratory, USA \\ ${ }^{7}$ ) Royal Institute of Technology, Sweden
}

\begin{abstract}
In Task A of the international DECOVALEX-THMC project, five research teams study the influence of thermal-hydro-mechanical (THM) coupling on the safety of a hypothetical geological repository for spent fuel. In order to improve the analyses, the teams calibrated their bentonite models with results from laboratory experiments, including swelling pressure tests, water uptake tests, thermally gradient tests, and the CEA mock-up THM experiment. This paper describes the mathematical models used by the teams, and compares the results of their calibrations with the experimental data.
\end{abstract}

\section{INTRODUCTION}

In Task A of the international DECOVALEX -THMC project, five research teams (Table 1) study the influence of thermal-hydro-mechanical (THM) coupling on the safety of the near-field of a hypothetical geological repository for spent fuel. The repository would be located in the Canadian Shield, with a horizontal drift geometry, and the copper container for the Candu fuel emplaced in the drift would be surrounded by MX- 80 bentonite. The task definition and the results of preliminary analyses are reported in a companion paper by Nguyen et al. (2006) in these proceedings. In order to refine the analyses in the next stages of Task A, the teams calibrated their bentonite models against results from laboratory experiments, including swelling pressure tests, water uptake tests, thermal gradient tests by SKB (Börgesson and Hernelind, 1999), and the CEA (Gatabin and Billaud, 2005) mock-up THM test. In the swelling pressure tests, samples of bentonite of different densities are re-saturated under confined conditions, and the swelling pressure is measured as a function of time. In the water uptake tests, the samples are supplied with water at one end, and the progress of re-saturation in the samples is monitored. In the thermal gradient tests, the samples are submitted to a thermal gradient, and the redistribution of moisture through the samples is monitored. The size of the samples for the above experiments is rather small $(5 \mathrm{~cm}$ in diameter and $5 \mathrm{~cm}$ in length). The CEA mock-up test is performed on a larger bentonite sample, $20 \mathrm{~cm}$ in diameter and $20 \mathrm{~cm}$ in length. The sample is submitted to thermal gradient along its length, and after a few months water is supplied at the bottom. Measurements of temperature, stresses and relative humidity were performed at many points. This paper describes the mathematical models used by the teams for simulating these tests and compares the results of their calibration with the experimental data.

Table 1 Research team and simulation code

\begin{tabular}{|l|l|}
\hline Research team & Simulation code \\
\hline \hline CNSC (Canada) & FRACON \\
\hline JAEA (Japan) & THAMES \\
\hline SKI (Sweden) & ROCMAS \\
\hline SKB (Sweden) & ABAQUS \\
\hline STUK (Finland) & ELMER \\
\hline
\end{tabular}

\section{OUTLINE OF THE LABORATORY TESTS \\ 2.1 Water uptake tests}

This test was conducted by confining samples with a known degree of saturation in stiff cylinders and applying a filter stone with zero water pressure at one end. The negative water pressure of the unsaturated sample sucked water into the sample. The test was brought to an end in different times for different samples. Each sample was then sliced into a number of sections and the water ratio of each section was determined. With this test the degree of saturation was plotted as a function of the distance from the water inlet. Figure 1 shows the results of the water uptake tests conducted with an initial void ratio of 0.75 and an initial degree of 
saturation $40 \%$.

\subsection{Thermal gradient tests}

These tests can be performed in a stiff oedometer with water-tight boundaries by applying a constant thermal gradient along the sample axis. The tests were finished after different times and the samples were sliced in the same way as in the previous test. Figure 2 shows the results of the thermal gradient test conducted with an initial void ratio of 1.0 and an initial degree of saturation $50 \%$.

\subsection{Swelling pressure test}

The swelling pressure was measured with the initial void ratio after completed saturation by confining samples in stiff cylinders.

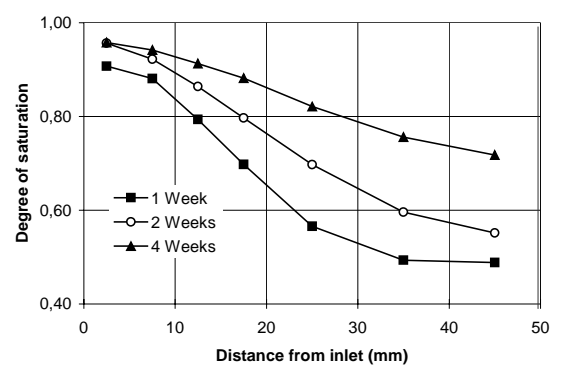

Figure 1 Result of the water uptake test

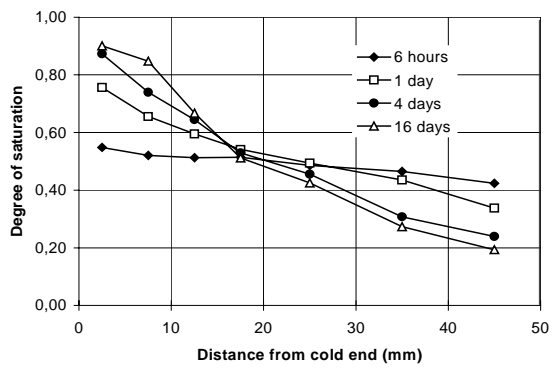

Figure 2 Result of the thermal gradient test

\subsection{CEA Mock-up test}

The THM mock-up test that has been performed on vertical cylindrical columns of compacted MX-80 bentonite was conducted by the Commissariat de l'Énergie Atomique (CEA) in France (Figure 3). The test was composed of two phases. In Phase 1 heat was applied to one end of the column while the temperature at the other end was kept constant and equal to $20^{\circ} \mathrm{C}$. A maximum temperature of $150^{\circ} \mathrm{C}$ was applied. Phase 2 started after thermal equilibrium had been achieved, followed by gradual hydration of the sample. A constant water pressure was applied to the end opposite to the one where the temperature variation was prescribed. Constant volume conditions were ensured in the two phases of the test. The samples had a diameter and a height of $203 \mathrm{~mm}$. The sample was tightly enclosed in a PTFE sleeve. To minimize heat losses, the cells were insulated with a heatproof envelope. The experiment was not gas tight. Heat was applied at the bottom plate whereas hydration proceeds from the top of the sample. The dry density of MX-80 in the mock-up test is different from that of the small scale laboratory tests. The dry density of MX- 80 by SKB test is $1.67 \mathrm{~g} / \mathrm{cm}^{3}$, whereas in the mock-up test it is approximately $1.8 \mathrm{~g} / \mathrm{m}^{3}$. The void ratio for the mock-up test is about 0.6 compared to 0.77 for the previous laboratory tests.

Temperature ( $\mathrm{T} 0$ to $\mathrm{T} 14$ ) and relative humidity (HR1 to HR7) are measured as shown in Figure 3. Figure 4 and Figure 5 shows the measured results.
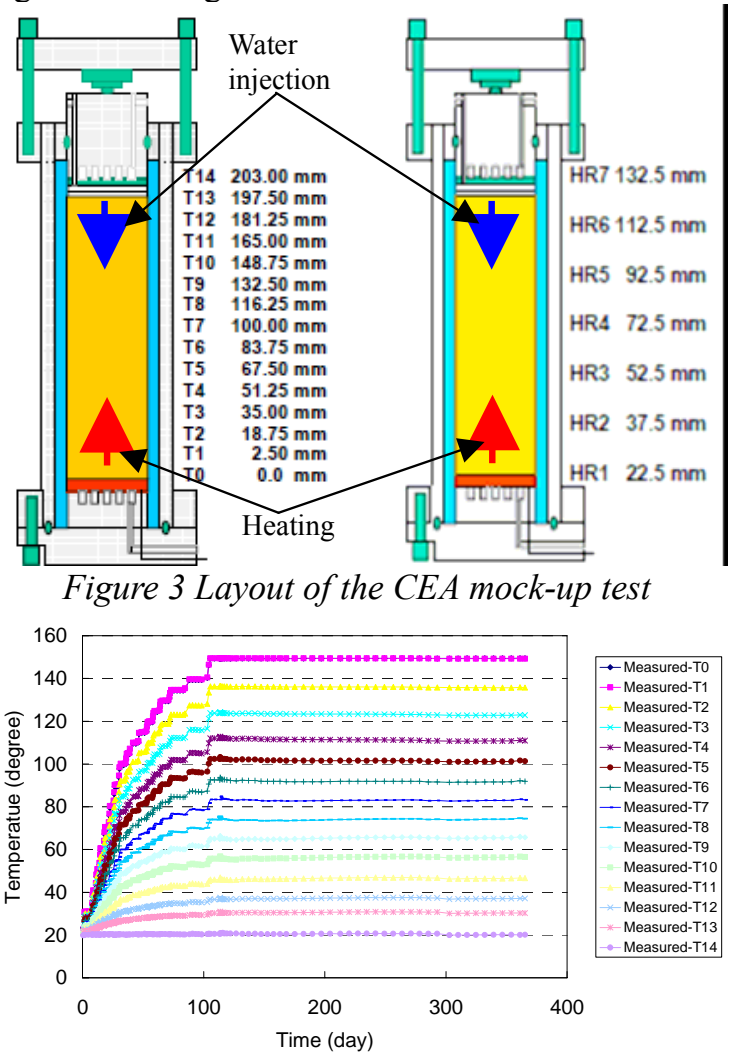

Figure 4 Measured temperatures in the CEA mock-up test

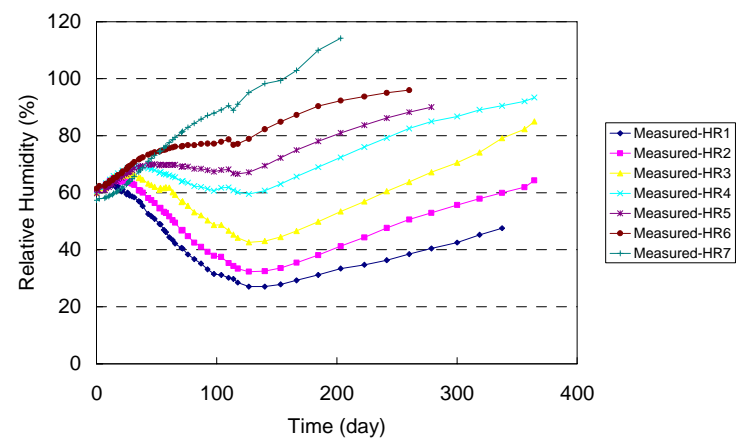

Figure 5 Measured relative humidity in the CEA mock-up test 


\section{PHYSICAL PROCESSES AND CONSTITUTIVE MODELS \\ 3.1 The CNSC model}

In the FRACON code (Rutqvist et al., 2001) developed and used by the CNSC, the bentonite was assumed to be a poroelastic continuum of a generalized Biot's type. The physical processes considered are the heat conduction, pore water flow in saturated /unsaturated porous media, vapor flow driven by temperature gradients, and mechanical deformation of the skeleton. These processes are described by the following governing equations.

The energy conservation equation is given as

$\frac{\partial}{\partial x_{i}}\left(\kappa_{i j} \frac{\partial T}{\partial x_{j}}\right)+q=\rho C \frac{\partial T}{\partial t}$

where $\mathrm{T}$ is temperature, $\kappa_{i j}$ is the heat conductivity tensor, $q$ the heat source (or sink), $\rho$ the bulk density and $C$ is the specific heat.

The continuity equation of groundwater is given as

$$
\begin{aligned}
& \frac{\partial}{\partial x_{i}}\left[\frac{\rho_{w} k_{i j} K_{r}}{\mu}\left(\frac{\partial P}{\partial x_{j}}+\rho_{w} g_{i}\right)\right]-\frac{\partial}{\partial x_{i}}\left(D_{T v} \frac{\partial T}{\partial x_{i}}\right) \\
& +\rho_{w} n \frac{d S_{r}}{d P} \frac{\partial P}{\partial t}-S_{r} B_{w} \frac{\partial P}{\partial t} \\
& -\rho_{w} S_{r} \frac{\partial}{\partial t}\left(\frac{\partial u_{r}}{\partial x_{r}}\right)+\rho_{w} S_{r}\left[n \beta_{w}+(1-n) \beta_{s}\right] \frac{\partial T}{\partial t}=0
\end{aligned}
$$

where $k_{i j}$ is the saturated permeability tensor; $K_{r}$ is the non-dimensional relative permeability of the unsaturated medium; $S_{r}$ the function of the degree of saturation; $\mu$ the water viscosity; $\rho_{w}$ the water density; $D_{T v}$ the thermal diffusivity of the vapor; $n$ the porosity; and $B_{w}$ the coefficient of water compressibility. The symbol $\beta_{w}$ and $\beta_{s}$ are the thermal expansion of water and solid, respectively. $P$ is the water pressure.

The equilibrium equation is given as

$G \frac{\partial^{2} u_{i}}{\partial x_{j} \partial x_{j}}+(G+\lambda) \frac{\partial^{2} u_{j}}{\partial x_{i} \partial x_{j}}+\chi(S) \frac{\partial P}{\partial x_{i}}-\beta K_{d} \frac{\partial T}{\partial x_{i}}+F_{i}=0$

where, $u_{i}$ is the displacement vector, $G$ and $\lambda$ are Lame's elasticity constants, $\chi$ is the parameter for the effective stress, $\beta$ is the volumetric thermal expansion coefficient of the solid matrix and $F_{i}$ is the body force. Using a state-surface equation approach, $G$ and $\lambda$ and $\chi$ are expressed as functions of void ratio and degree of saturation (Nguyen et al., 2005)

\subsection{The JAEA model}

The behavior of the buffer material is influenced by the interdependence of thermal, hydraulic and mechanical phenomena. To simulate the water/vapor movement and heat induced water movement, the continuity equation used in the extended THAMES code (Rutqvist et al., 2001) is as follows;

$$
\begin{aligned}
& \left\{\xi \rho_{w} D_{\theta} \frac{\partial \theta}{\partial \psi}\left(h_{, i}-z, i\right)+(1-\xi) \frac{\rho_{w}{ }^{2} g K}{\mu_{l}} h_{, i}\right\}+\left\{\rho_{w} D_{T v} T_{i, i}\right\}_{i} \\
& -\rho_{w o} n S_{r} \rho_{w} g \beta_{P} \frac{\partial h}{\partial t}-\rho_{w} \frac{\partial \theta}{\partial \psi} \frac{\partial h}{\partial t}-\rho_{w} S_{r} \frac{\partial u_{i, i}}{\partial t}+\rho_{w o} n S_{r} \beta_{T} \frac{\partial T}{\partial t}=0
\end{aligned}
$$

where $D_{\theta}$ is the isothermal water diffusivity, $\theta$ the volumetric water content, $\psi$ the water potential and $K$ the intrinsic permeability. The symbol $\xi$ is the relative saturation parameter so that $\xi=0$ when the medium is fully saturated, $\xi=1$ otherwise. $\mu_{l}$ is the viscosity of water, $n$ the porosity, $S_{r}$ the degree of saturation, $\beta_{P}$ the compressibility of water, $\beta_{T}$ the thermal expansion coefficient of water and $z$ the elevation head. The variable $u_{i}$ is the displacement vector, $T$ is temperature, $h$ is the total head and $t$ is time. The subscript 0 means the reference state.

The energy conservation equation takes into account of the heat transfer by conduction and convection, as well as the energy change by evaporation. The equation is given as

$$
\begin{aligned}
& \left(\rho C_{v}\right)_{m} \frac{\partial T}{\partial t}+n S_{r} \rho_{w} C_{v l} V_{l i} T_{, i}-K_{T m} T_{, i i}+L\left\{D_{\theta v} \frac{\partial \theta}{\partial \psi}\left(h_{i}-z, i\right)\right\}, i \\
& +n S_{r} T \frac{\beta_{T}}{\beta_{P}}\left\{\xi D_{\theta} \frac{\partial \theta}{\partial \psi}\left(h_{i, i}-z, i\right)+(1-\xi) \frac{\rho_{w} g K}{\mu_{l}} h_{, i}+D_{T} T, i,\right\}_{i}, \\
& +\frac{1}{2}(1-n) \beta T \frac{\partial}{\partial t}\left(u_{i, j}+u_{j, i}\right) \delta_{i j}=0
\end{aligned}
$$

where $\left(\rho C_{v}\right)_{m}$ is the specific heat of the material consisting of water and the solid particles, $C_{v l}$ the specific heat of water, $V_{l i}$ the velocity vector of water, $K_{T m}$ the thermal conductivity of the medium consisting of water and the solid particles, $L$ the latent heat of vaporization per unit volume, $D_{\theta_{v}}$ the vapor diffusivity.

The equilibrium equation takes the swelling behavior of the partially saturated bentonite into account.

$\left[\frac{1}{2} C_{i j k l}\left(u_{k, l}+u_{l, k}\right)-F \pi \delta_{i j}-\beta \delta_{i j}\left(T-T_{o}\right)+\chi \delta_{i j} \rho_{w} g(h-z)\right]+\rho b_{i}=0$

where $C_{i j k l}$ is the elastic matrix, $\rho$ the density of the medium and $b_{i}$ the body force. $\chi$ is the parameter for the effective stress, $\chi=0$ for the unsaturated zone and $\chi=1$ for the saturated zone. The symbol $F$ is the coefficient relating to the swelling pressure process and $\beta=(3 G+2 \lambda) \alpha_{s}$, where $\alpha_{s}$ is the thermal expansion coefficient.

\subsection{The SKI model}

The governing equations of ROCMAS (Rutqvist et al., 2001) are as follows. The mass conservation of water and vapor, energy conservation and momentum conservation equations are given as

$$
\begin{aligned}
& { }_{\alpha}\left[S_{r} \rho_{w}+\left(1-S_{r}\right) \rho_{v}\right]+\left[n S_{r} \rho_{w}^{0} \beta_{p}+n\left(\rho_{w}-\rho_{v}\right) C_{s}+n\left(1-S_{r}\right) \frac{\rho_{v}}{\rho_{w} R T}\right] \frac{\partial P}{\partial T} \\
& +\left[n S_{r} \rho_{w}^{0} \beta_{T}+n\left(1-S_{r}\right)\left(h \frac{\partial \rho_{v s}}{\partial T}+\frac{\rho_{v} P}{R T^{2}}\right)\right] \frac{\partial T}{\partial t} \\
& =\nabla \cdot\left[\left(\frac{\rho_{w} k_{w}}{\mu_{w}}+D_{P_{v}}\right) \nabla P+\frac{\rho_{w} k_{w}}{\mu_{w}} \gamma_{w} \nabla z\right]-\nabla \cdot\left(D_{T v} \nabla T\right)
\end{aligned}
$$




$$
\begin{aligned}
& {\left[(1-n) \rho_{s} C_{v s}+\rho_{w} C_{w s} n S_{r}+\rho_{v} C_{p v} n\left(1-S_{r}\right)+H_{l}\left(h \frac{\partial \rho_{v s}}{\partial T}-\frac{\rho_{v} P}{R T^{2}}\right)\right] \frac{\partial T}{\partial t}} \\
& +\beta T_{0} \frac{\partial e}{\partial t}+\nabla \cdot\left(C_{v w} q_{w}^{m}+C_{p v} q_{v}^{m}\right) \nabla T=\nabla \cdot\left[\left(K_{M}+L D_{T v}\right) \cdot \nabla T+L D_{p v} \nabla P\right] \\
& -H_{2} \frac{\partial\left(n S_{r}\right)}{\partial t}-\left(H_{1} \frac{\rho_{v}}{\rho_{w} R T}+H_{2} \frac{\partial\left(n S_{r}\right)}{\partial P}\right) \frac{\partial P}{\partial t} \\
& \frac{\partial}{\partial x_{j}}\left[C_{i j k l} e_{k l}-\delta_{i j} \beta\left(T-T_{0}\right)-\delta_{i j} \zeta\left(S_{r}-S_{r 0}\right)-\delta_{i j} \alpha S_{r} \bar{P}\right]+\bar{\rho}_{s} f=0
\end{aligned}
$$

where, $\alpha$ is the Biot's coupling constant, $\gamma_{w}$ the fluid density by weight, $\xi$ the coefficient of swelling, the average $\bar{\rho}$ solid mass density, $\rho_{v}$ the vapor mass density, $\rho_{v s}$ the saturated vapor mass density, $C_{s}$ the moisture capacity, $e$ the volumetric elastic strain, $R$ the specific gas constant, $f$ the body force per unit mass, $k_{w}$ the water permeability, $K_{M}$ the thermal conductivity of solid-fluid mixture, $D_{v}$ the effective molecular diffusivity of water vapor in air, $L$ the latent heat of vaporization, and the $C_{v s}$, $C_{v w}, C_{P v}$ the volumetric specific heat capacity of solid, water and vapor, respectively.

\subsection{The SKB model}

The finite element code ABAQUS (see e.g. Börgesson and Hernelind, 1999 or Rutqvist et al, 2001) was used for the calculations. ABAQUS contains a capability of modelling a large range of processes in many different materials as well as complicated three-dimensional geometry. The code includes special material models for rocks and soils and ability to model geological formations with infinite boundaries and in situ stresses by e.g. the self-weight of the medium. It also includes a capability to make substructures with completely different finite element meshes and mesh densities. The hydro-mechanical model consists of porous medium and wetting fluid and is based on equilibrium equations, constitutive equations, energy balance equations and mass conservation equations using the effective stress theory. Equilibrium is expressed by writing the principle of virtual work for the volume under consideration in its current configuration at time $t$ :

$\int_{\mathrm{V}} \boldsymbol{\sigma}: \delta \boldsymbol{\varepsilon} d V=\int_{\mathrm{s}} \mathbf{t} \cdot \delta \mathbf{v} d S+\int_{\mathrm{V}} \mathbf{f} \cdot \delta \mathbf{v} d V+\int_{\mathrm{V}} S_{r} n \rho_{w} \mathbf{g} \cdot \delta \mathbf{v} d V$,

where $\delta \mathbf{v}$ is a virtual velocity field, $\delta \varepsilon$ is the virtual rate of deformation, $\sigma$ is the true (Cauchy) stress, $\mathbf{t}$ are the surface tractions per unit area, $\mathbf{f}$ are all body forces except the weight of the wetting liquid, $S_{r}$ is the degree of saturation, $n$ the porosity, $\rho_{\mathrm{w}}$ the density of the wetting liquid and $\mathbf{g}$ is the gravitational acceleration.

The mass continuity equation for the fluid combined with the divergence theorem implies the pointwise equation:

$\frac{1}{J} \frac{d}{d t}\left(J \rho_{\mathrm{w}} S_{r} n\right)+\frac{\partial}{\partial \mathbf{x}} \cdot\left(\rho_{\mathrm{w}}\left(-\hat{\mathbf{k}} \frac{\partial \phi}{\partial \mathbf{x}}\right)\right)=0$.

where $J$ is the determinant of the Jacobian matrix of the skeleton motion and $\mathbf{x}$ is the position. The constitutive behaviour for pore fluid is governed by Darcy's law, which is generally applicable to low fluid velocities. Darcy's law states that, under uniform conditions, the volumetric flow rate of the wetting liquid through a unit area of the medium. $\hat{\mathbf{k}}$ is the permeability of the medium and $\phi$ is the piezometric head. Vapour flow is modelled as a diffusion process driven by a temperature gradient

The basic energy balance of heat transfer is

$\int_{\mathrm{V}} \rho \dot{\mathrm{U}} \mathrm{dV}=\int_{\mathrm{S}} \mathrm{qdS}+\int_{\mathrm{V}} \mathrm{rdV}$

where $V$ is the volume of solid material with a surface area $S$; $\rho$ is the density of the material; $\dot{U}$ is the material time rate of the internal energy; $q$ is the heat flux per unit area of the body, flowing into the body; and $r$ is the heat supplied externally into the body per unit volume.

\subsection{The STUK model}

The numerical calculations were performed with ELMER (CSC, 2005), which is a software package for solving coupled partial differential equations generated by multiphysics problems in continuum mechanics. The program was developed at the Finnish IT center for science CSC in collaboration with Finnish universities, research centers and industry. A particular form of the program is being created in cooperation with the experts of CSC to solve the problems presented here.

The final primary variables are porosity $\eta$, gaseous pressure $\hat{B}$, saturation $\chi$, solid phase displacement $\mathbf{u}=\mathbf{u}_{\mathrm{s}}$, and temperature $T$. Inertial and convectional terms are neglected. The conservation equations for solid mass, air mass, water mass, momentum, and energy are reduced to

$$
\begin{aligned}
& \frac{\partial \rho_{\mathrm{s}}}{\partial t}+\nabla \cdot\left(\rho_{\mathrm{s}} \frac{\partial \mathbf{u}}{\partial t}\right)=0, \\
& \frac{\partial \rho_{\mathrm{a}}}{\partial t}+\nabla \cdot\left(\rho_{\mathrm{a}} \mathbf{V}_{\mathrm{a}}+\rho_{\mathrm{a}} \frac{\partial \mathbf{u}}{\partial t}\right)=0, \\
& \frac{\partial\left(\rho_{1}+\rho_{\mathrm{v}}\right)}{\partial t}+\nabla \cdot\left(\rho_{1} \mathbf{V}_{1}+\rho_{\mathrm{v}} \mathbf{V}_{\mathrm{v}}+\left(\rho_{1}+\rho_{\mathrm{v}}\right) \frac{\partial \mathbf{u}}{\partial t}\right)=0, \\
& \nabla \cdot \boldsymbol{\sigma}+\rho \mathbf{g}=0, \\
& (\rho c)_{\text {eff }} \frac{\partial T}{\partial t}-\left(e_{\mathrm{v}}-e_{1}\right) \theta_{1}+\nabla \cdot \mathbf{q}-Q=0,
\end{aligned}
$$

respectively, where $\rho_{k}$ is apparent constituent density, $\mathbf{V}_{\mathrm{k}}$ is relative constituent velocity, $\boldsymbol{\sigma}$ is the Cauchy stress, $e_{\mathrm{k}}$ is the specific constituent internal energy, $\theta_{1}$ is the rate of liquid mass production, $\mathbf{g}$ is the gravitational vector, and $Q$ is the source of energy. The general model is described in (Jussila (2006a, 2006b), Jussila and Ruokolainen (2005)) and the specific constitutive equations in (Jussila (2006b), Jussila and Ruokolainen (2005)).

\section{COMPARISON OF RESULTS}

Figure 6 compares the simulation results of the 
water uptake test by five models. After 1 week from the start of infiltration, the simulation results by all teams except for STUK who assumed a higher initial saturation shows reasonably good agreements with the measured results (Figure 6a). After 4 weeks, SKI and JAEA obtain good agreements with the measured result, whereas the CNSC, STUK and SKB overpredict the measured infiltration rate (Figure 6b). Figure 7 compares the simulation results of the thermal gradient test. After 1 day, good agreements between simulated and measured results are obtained in the wetting region. However, for the drying region the simulation results generally indicate less drying than measured, except for the results of the SKI team, whose simulation results are very close to the measured results (Figure 7a). Similar tendency is noted after 16 days (Figure 7b). Furthermore, the observed total mass of water is less than all simulation results. This is because of the initially lower saturation assumed in the simulation and possible water leaking out of the oedometer by evaporation of water from the specimen during long-term experimental periods. Figure 8 compares the simulation results of the swelling pressure test. Maximum swelling pressure is between 6 and $9 \mathrm{MPa}$ and it becomes constant after approximately $6.0 \mathrm{E} 6 \mathrm{sec}$. The measured values for a dry density of $1.67 \mathrm{~g} / \mathrm{cm}^{3}$ was about 6 to $8 \mathrm{MPa}$

Figure 9 presents the comparison of the simulation results of the CEA mock-up test. The temperature is well matched and stays approximately constant during phase 2 in both experiments and simulations (Figure 9a). However, at point T5, simulation results by SKI and SKB overpredict the measured temperature, whereas JAEA and STUK underpredict the measured temperature. The general evolution of relative humidity agrees quite well except for the JAEA result (Figure 9b). The experiments and the simulations results show similar general behaviors with a drying to about $30 \%$ and $40 \%$ during phase 1 , respectively, and then a gradual wetting during phase 2. The rate of the gradual wetting during phase 2 is controlled by permeability. The discrepancy in JAEA's results is caused by the use of an inappropriate water retention curve leading to a relatively high humidity. Therefore, the initial relative humidity by JAEA is quite higher than the measured data. The general evolution and magnitude of axial stress is in general agreement between models and experiments, with a maximum stress of about 10MPa (Figure 9c).

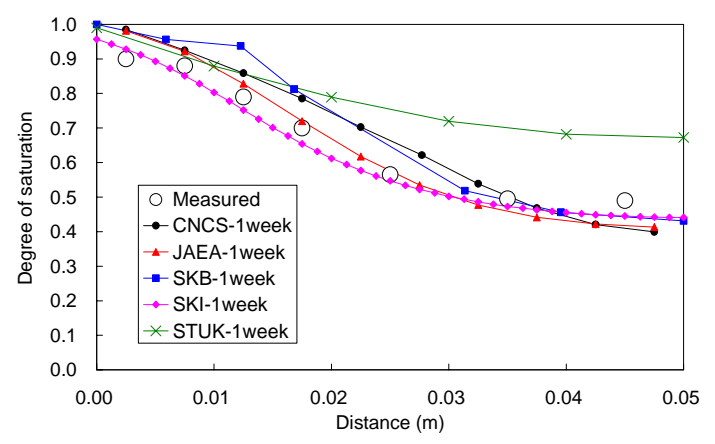

(a) After 1 week

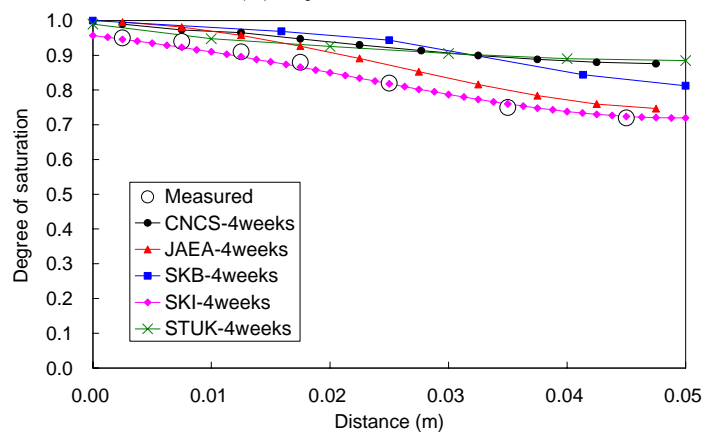

(b) After 4 weeks

Figure 6 Comparison of the water uptake test

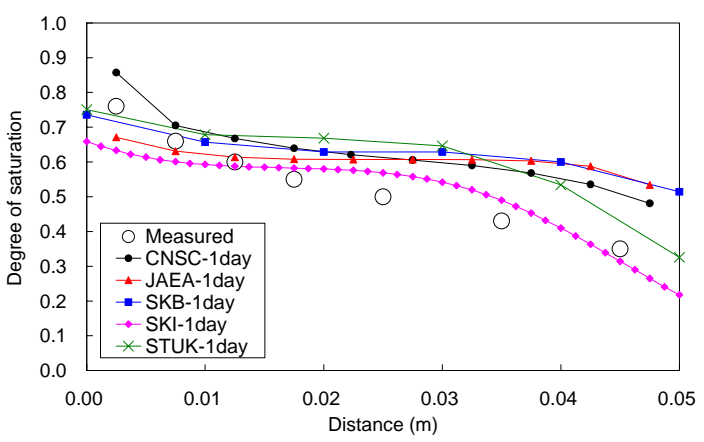

(a) After 1 day

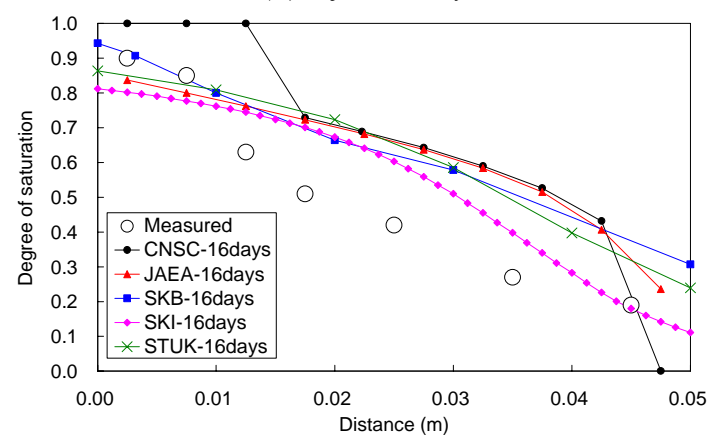

(b) After 16 days

Figure 7 Comparison of the thermal gradient test 


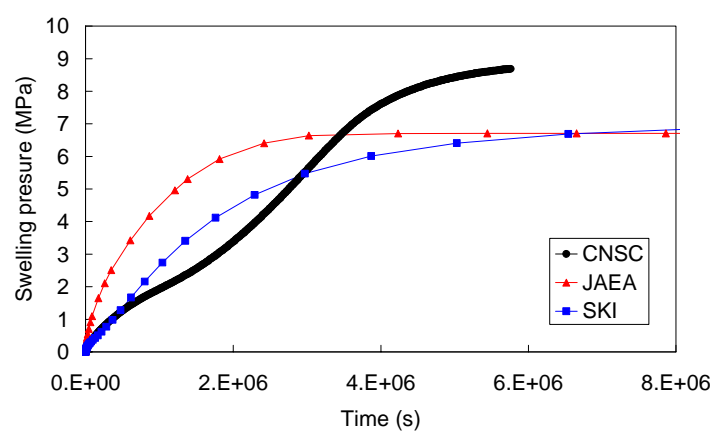

Figure 8 Comparison of the swelling pressure test

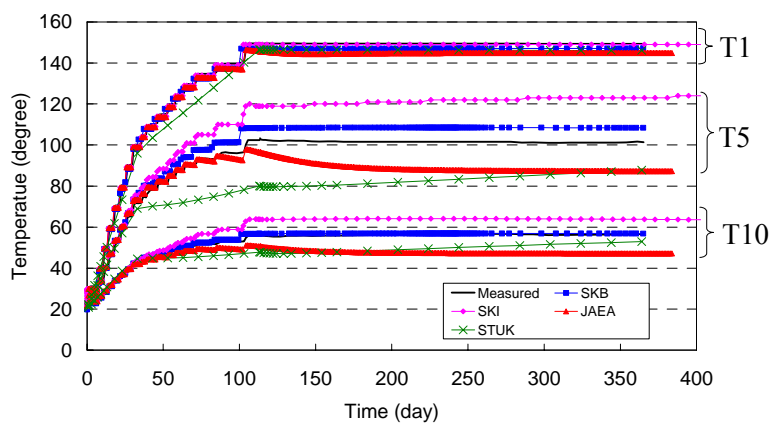

(a) Temperature

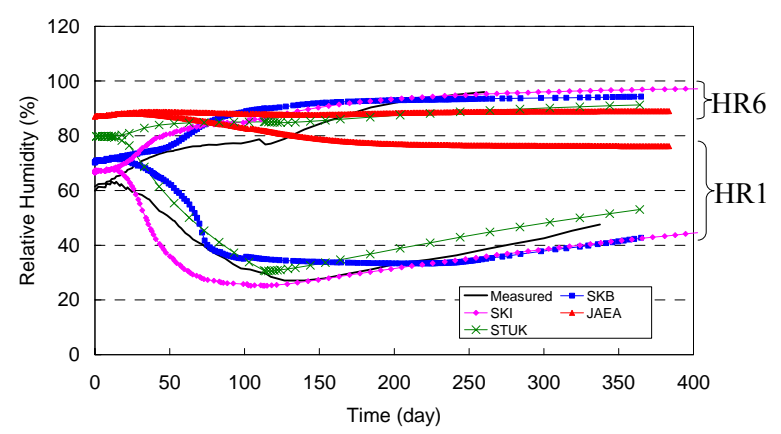

(b) Relative humidity

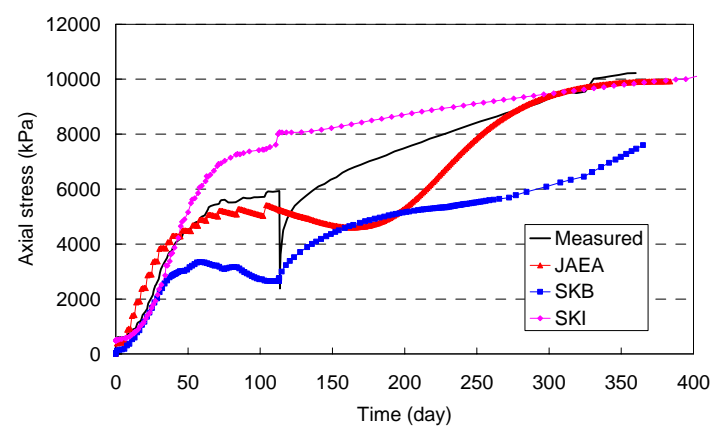

(c) Axial stress

Figure 9 Compoarison of the CEA mock-up test

\section{SUMMARY}

Calibrations of models of five teams were performed by numerical simulation of the small-scale laboratory tests by SKB and a large-scale laboratory THM test by CEA. Parameters for unsaturated water movement under constant temperature, vapor flow due to thermal gradient and swelling pressure were calibrated. In order to obtain a better quantitative agreement, some teams might go into second calibration iteration before applying their models for a final THM analysis of the hypothetical repository.

\section{ACKNOWLEDGEMENTS}

Funding to the LBNL research team and was provided by the Swedish Nuclear Waste Power Inspectorate (SKI). This work was, in part, supported by the U.S. Dept. of Energy under Contract No. DE-AC02-05CH11231.

\section{REFERENCES}

Börgesson L. and Hernelind J.: Coupled thermo-hydro-mechanical calculations of the water saturation phase of a KBS-3 deposition hole, Swedish Nuclear Fuel and Waste Management Co., Technical Report TR-99-41, 1999.

CSC: The Finnish IT Center for Science: ELMER [WWW document], http://www.csc.fi/Elmer/

Gatabin C. and Billaud P.: Bentonite THM mock-up experiments. Sensors data report, CEA, France, 2005.

Jussila, P.: Thermomechanics of Porous Media I: Thermohydraulic Model for Compacted Bentonite, Transport Porous Med, 62, 81-107, 2006a.

Jussila, P.: Thermomechanics of swelling unsaturated porous media - compacted bentonite clay in spent fuel disposal, Doctorate thesis, under preparation, Helsinki University of Technology, 2006b.

Jussila, P. and Ruokolainen, J.: Thermomechanics of Porous Media II: THM Model for Compacted Bentonite, Transport Porous Med, submitted for publication, 2005.

Nguyen, T.S., Selvadurai, A.P.S. and Armand G,: Modelling the FEBEX THM experiment using a state surface approach. Int.J. of Rock Mechanics and Mining Sciences, Vol. 42, No.5-6, pp.639-651, 2005.

Nguyen, T.S., Börgesson, L., Chijimatsu, M., Fujita, T., Hernelind, J., Jussila, P., Rutqvist, J. and Jing, L.: Influence of coupled THM phenomena on the safety of a spent fuel repository: A near-field study, GeoProc 2006, 2006.

Rutqvist, J., Börgesson, L., Chijimatsu, M., Kobayashi, A., Jing. L., Nguyen, T.S., Noorishad. 
J. and Tsang, C.-F.: Thermohydromechanics of partially saturated geological media: governing equations and formulation of four finite element models. Int.J. of Rock Mechanics and Mining Sciences, Vol. 38, No.1, pp.105-128, 2001. 